doi:10.1016/j.ijantimicag.2006.05.027

Copyright (c) 2006 Elsevier B.V. and the International Society of Chemotherapy All rights reserved.

\title{
Antibiotic resistance, integrons and Salmonella genomic island 1 among non-typhoidal Salmonella serovars in The Netherlands
}

\section{An T.T. Vo ${ }^{a, b}$, Engeline van Duijkeren ${ }^{a}, *$, Ad C. Fluit ${ }^{c}$, Wim J.B. Wannet ${ }^{d}$, Anjo J. Verbruggen ${ }^{d}$, Henny M.E. Maas ${ }^{d}$ and Wim Gaastra ${ }^{a}$}

${ }^{a}$ Department of Infectious Diseases and Immunology, Faculty of Veterinary Medicine, Utrecht University, P.O. Box 80165, 3508 TD Utrecht, The Netherlands

${ }^{\mathrm{b}}$ Faculty of Animal Science and Veterinary Medicine, NongLam University, Vietnam ${ }^{c}$ Eijkman-Winkler Institute, University Medical Center Utrecht, Utrecht University, Utrecht, The Netherlands

${ }^{\mathrm{d} N a t i o n a l}$ Institute of Public Health and the Environment, Bilthoven, The Netherlands

Received 1 February 2006; accepted 19 May 2006. Available online 17 August 2006.

\section{Abstract}

The objective of this study was to investigate the antimicrobial resistance patterns, integron characteristics and gene cassettes as well as the presence of Salmonella genomic island 1 (SGI1) in non-typhoidal Salmonella (NTS) isolates from human and animal origin. Epidemiologically unrelated Dutch NTS strains ( $n=237$ ) originating from food-producing animals and human cases of salmonellosis were tested for their susceptibility to 15 antimicrobial agents. Resistance to 14 of these antimicrobials, including the third-generation cephalosporins, was detected. Resistance to sulphonamides, ampicillin, tetracycline, streptomycin, trimethoprim and nalidixic acid was common $(\geq 10 \%$ of the strains were resistant). Resistance against three or more antimicrobials was observed in 57 isolates. The same 237 strains were studied for the prevalence of class 1 integrons, their gene cassettes and the presence of SGI1. Thirty-six isolates (15.2\%) carried class 1 integrons. These integrons had ten distinct profiles based on the size of the integron and restriction fragment length polymorphism analysis. Integrons were detected for the first time in serovars Indiana and Senftenberg. Multidrug resistance was strongly associated with the presence of class 1 integrons in which the aadA2, aadA1, bla PSE-1, dfrA1, dfrA5, dfrA14 or sat genes were present, as determined by nucleotide sequence determination. The presence of gene cassettes or combinations of gene cassettes not previously found in integrons in Salmonella was observed. SGI1 or its variants (SGI-B, -C and -F) were present in 16 isolates belonging to either serovar Typhimurium, Derby or Albany. Regardless of whether the isolate was of human or animal origin, the same resistance phenotype, integron profile and SGI1 structure could be observed.

Keywords: Antimicrobial resistance; Integrons; Salmonella; Salmonella genomic island 1 


\section{Introduction}

Salmonellosis is a major zoonotic disease in humans, causing $68 \%$ of the outbreaks of food-borne diseases reported in Europe between 1993 and 1998. In The Netherlands, 50000 cases of salmonellosis are reported each year [1]. The majority of the 2500 Salmonella serovars can cause food-borne salmonellosis in humans [2].

Antimicrobial resistance in Salmonella spp. is a major health problem in human and veterinary medicine worldwide [3]. Many antimicrobial resistance genes are associated with genetic elements called integrons [4], which can be located on transposons and plasmids but also on the chromosome. They are able to integrate and express genes coding for antibiotic resistance [5] and [6]. Class 1 integrons, the most common integron type [5], have been detected in many countries in different Salmonella serovars [7] and may be located on the so-called Salmonella genomic island 1 (SGI1) [8], [9], [10], [11] and [12]. SGI1 is an integrative $43 \mathrm{~kb}$ mobilisable chromosomal element [13] on which antibiotic resistance genes are clustered, flanked by two class 1 integrons [14], [15] and [16]. Strains containing SGI1 are usually resistant to ampicillin (and amoxicillin), streptomycin, spectinomycin, chloramphenicol (and florfenicol), sulphonamides and tetracycline. Strains carrying SGI1 variants (SGI1-A to SGI1-J) with different antibiotic resistance profiles have also been found in several Salmonella serovars [9], [11], [12] and [17]. It is important to study the spread of antibiotic resistance to understand the relationship between antibiotic resistance genes, class 1 integrons and SGI1, as integrons and transferable elements are responsible for today's spread of resistance genes in the bacterial population and increase the overall resistance gene pool.

The aim of the present study was to determine (1) the antimicrobial resistance profiles of Salmonellae isolated from humans, cattle, pigs, chickens and food products in The Netherlands, (2) the prevalence and molecular characteristics of class 1 integrons in these strains and (3) the genetic basis of SGI1.

\section{Materials and methods}

\subsection{Bacteria}

The 237 epidemiologically unrelated Salmonella isolates in this study were derived from a collection of 3265 isolates obtained in 2004 by the Dutch National Institute of Public Health and the Environment (RIVM) and the Veterinary Microbiological Diagnostic Center of Utrecht University. All isolates were confirmed to be Salmonella based on colony morphology and biochemical tests [18]. The isolates in this study were chosen to represent different serotypes and different origins of isolation, i.e. humans $(n=114)$, cattle $(n=44)$, pigs $(n=24)$, chickens $(n=47)$ and food products (meat or eggs; $n=8)$. All human isolates were from clinical cases. Of the animal isolates, $73 \%$ were from clinical cases (mainly cattle and pigs) and $17 \%$ were from healthy animals (mostly chickens). The isolates were serotyped according to the latest version of the Kauffmann-White scheme using slide and microtitre plate agglutination [19]. Serovar Enteritidis isolates were phage-typed as described by Ward et al. [20]. Serovar Typhimurium isolates were phage-typed using the Dutch phage typing system [21].

\subsection{Antimicrobial susceptibility}


Antimicrobial susceptibility was determined by the agar diffusion technique using commercial disks (Oxoid Ltd., Basingstoke, UK) according to the guidelines of the Clinical and Laboratory Standards Institute (formerly the National Committee for Clinical Laboratory Standards) [22]. The antimicrobials tested were: ampicillin $10 \mu \mathrm{g}$, amoxicillin/clavulanic acid $30 \mu \mathrm{g}$, cefalothin $30 \mu \mathrm{g}$, ceftazidime $30 \mu \mathrm{g}$, chloramphenicol $30 \mu \mathrm{g}$, ciprofloxacin $5 \mu \mathrm{g}$, colistin $10 \mu \mathrm{g}$, gentamicin $10 \mu \mathrm{g}$, kanamycin $30 \mu \mathrm{g}$, nalidixic acid $30 \mu \mathrm{g}$, norfloxacin $10 \mu \mathrm{g}$, streptomycin $10 \mu \mathrm{g}$, tetracycline $30 \mu \mathrm{g}$, trimethoprim $5 \mu \mathrm{g}$ and sulphonamides $300 \mu \mathrm{g}$. Escherichia coli 25922 was used as the control organism.

\subsection{Detection of class 1 integrons}

Template DNA of all 237 isolates was prepared by the boiled lysate procedure [23]. Integrons were detected by polymerase chain reaction (PCR) amplification of the class 1 integrase-specific int 1 gene [24]. Subsequently, the size of any inserted gene cassette of the integrase-positive isolates was determined by PCR using primers for the conserved segment regions (CS-PCR) [23]. Since the 3' segment of the class 1 integron is not always conserved, some integrase-positive isolates yielded no product in the CS-PCR. In this case, the gene cassette of the isolate was characterised by an inverted PCR for the integrase gene. Briefly, $1 \mu \mathrm{g}$ of genomic DNA was cleaved with the restriction endonuclease SphI. The DNA obtained in this way was ligated and subjected to PCR using the int-OUT and CS-F primers (Table 1 ). Only amplicons of ca. $1 \mathrm{~kb}$ were sequenced. CS-PCR products were resolved by electrophoresis at $100 \mathrm{~V}$ for $3 \mathrm{~h}$ on $0.7 \%$ agarose gels containing ethidium bromide and visualised under ultraviolet light. 
Table 1.

Primers used for polymerase chain reaction

\begin{tabular}{|l|l|l|l|l|l|l|}
\hline Primer & Gene & Fragment amplification & Product size & Sequence (5'-3') & Reference \\
\hline int1-F & int1 & int1 & 242 & TCT CGG GTA ACA TCA AGG & Leverstein et al., 2002 [24] \\
\hline int1-R & int1 & & & AGG AGA TCC GAA GAC CTC & Leverstein et al., 2002 [24] \\
\hline int-OUT & int1 & Cassette(s) & a & AAG TGG TTC GCA TCC TCG & This study \\
\hline CS-F & Conserved segment & Cassette(s) & a & GGC ATC CAA GCA GCA AG & Levesque et al., 1995 [23] \\
\hline CS-R & Conserved segment & & & AAG CAG ACT TGA CCT GA & Levesque et al., 1995 [23] \\
\hline U7-L12 & thdf & Left junction & 500 & ACA CCT TGA GCA GGG CAA G & Doublet et al., 2003 [12] \\
\hline LJ-R1 & int1 & & & AGT TCT AAA GGT TCG TAG TCG & Doublet et al., 2003 [12] \\
\hline 104-R] & S044 & Right junction & 515 & TGA CGA GCT GAA GCG AAT TG & Doublet et al., 2003 [12] \\
\hline C9-L2 & int2 & & & AGC AAG TGT GCG TAA TTT GG & Doublet et al., 2003 [12] \\
\hline 104-D & yidY & dfrA1..orfC & 1057 & CCA GCA GCA AGC GCG TTA CG & Doublet et al., 2003 [12] \\
\hline dfrA1-F & dfrA1 & & & TCT CGA ATC AAG CAG GAA CC & This study \\
\hline orfC-R & orfC & floR & 494 & TTT GGW CCG CTM TCR GAC & This study \\
\hline cml01 & floR & & & SGA GAA RAA GAC GAA GAA G & Doublet et al., 2003 [12] \\
\hline cml15 & floR & intI..aadA2 & 1135 & GCT CTC GGG TAA CAT CAA GG & Doublet et al., 2003 [12] \\
\hline int1 & intI1 & & & GAC CTA CCA AGG CAA CGC TA & Doublet et al., 2003 [12] \\
\hline aad & aadA2 & sulI..floR & AAG GAT TTC CTG ACC CTG & Doublet et al., 2003 [12] \\
\hline sulTER & sul1delta & & AAA GGA GCC ATC AGC AGC AG & Doublet et al., 2003 [12] \\
\hline F3 & floR & & & Doublet et al., 2003 [12] \\
\hline
\end{tabular}




\begin{tabular}{|c|c|c|c|c|c|}
\hline Primer & Gene & Fragment amplification & Product size & Sequence $\left(5^{\prime}-3^{\prime}\right)$ & Reference \\
\hline F4 & floR & floR..tetR & 598 & ТТС СТС АСС ТТС АТС СТА СС & Doublet et al., 2003 [12] \\
\hline F6 & tetR & & & TTG GAA CAG ACG GCA TGG & Doublet et al., 2003 [12] \\
\hline tetR & tetR & tetR..tet $A$ & 1559 & GCC GTC CCG ATA AGA GAG CA & Doublet et al., 2003 [12] \\
\hline tetA & tet $A$ & & & GAA GTT GCG AAT GGT CTG CG & Doublet et al., 2003 [12] \\
\hline int2 & groEL-intI1 & int1..pse 1 & 1338 & TTC TGG TCT TCG TTG ATG CC & Doublet et al., 2003 [12] \\
\hline pse1 & pse-1 & & & САT CAT TTC GCT CTG CCA TT & Doublet et al., 2003 [12] \\
\hline pse-L & pse-1 & pse1..S044 & 4400 & AAT GGC AAT CAG CGC TTC CC & Doublet et al., 2003 [12] \\
\hline MDR-B & S044 & & & GAA TCC GAC AGC CAA CGT TCC & Doublet et al., 2003 [12] \\
\hline TEM-F & $b / a_{\mathrm{TEM}}$ & $b l a_{\mathrm{TEM}}$ & & ATG AGT ATT CAA CAT TTC CGT GTC G & This study \\
\hline TEM-R & bla & & & ACC AAT GCT TAA TCA GTG AGG CA & This study \\
\hline TEM-S1 & $b / a_{\mathrm{TEM}}$ & For sequencing & & ACA ACG ATC GGA GGA CCG & This study \\
\hline TEM-S2 & bla & For sequencing & & GCG GTT AGC TCC TTC GGT & This study \\
\hline SHV-F & $b^{\prime} a_{\mathrm{SHV}}$ & $b a_{\mathrm{SHV}}$ & & GTA TTG AAT TCA TGC GTT ATA TTC GCC TGT GTA & Bradford, 1999 [25] \\
\hline SHV-R & $b^{\prime} a_{\mathrm{SHV}}$ & & & CAG AAT TCG GCT AGC GTT GCC AGT GCT CGA & Bradford, 1999 [25] \\
\hline CTX-F & bla $a_{\text {стх-м }}$ & bla ${ }_{\mathrm{CTX}-\mathrm{M}}$ & 538 & CGA TGT GCA GTA CCA GTAA & A. Paauw, $2006^{\text {b }}$ \\
\hline CTX-R & bla $a_{\text {CTX-м }}$ & & & ATA TCG TTG GTG GTG CC & A. Paauw, $2006^{\mathrm{b}}$ \\
\hline
\end{tabular}

a Size depending on the gene cassette(s) inserted.

${ }^{b}$ Personal communication. 


\subsection{Gene cassette characterisation}

CS-PCR amplicons of the same size were restricted with two restriction endonucleases and were considered identical if they had the same restriction fragment length polymorphism (RFLP) pattern after digestion with both enzymes (Table 2). One sample of each representative RFLP type was randomly chosen for sequencing. In the case of a unique integron, purified CS-PCR products were cloned in the pGEM-T easy Vector (Promega, Madison, WI). Colonies containing plasmids with the inserted fragment were picked from Luria-Bertani plates containing ampicillin $(100 \mu \mathrm{g} / \mathrm{mL}), 40 \mu \mathrm{L}$ of IPTG (100 mM per plate) and $40 \mu \mathrm{L}$ of $\mathrm{X}-\mathrm{Gal}$ ( $2 \%$ per plate). The target fragments were obtained by PCR using T7 and Sp6 primers under the same conditions as described for the CS-PCR. The amplification products were purified using the Qia Gel Extraction kit (Qiagen, Hilden, Germany) and the nucleotide sequence was determined. For sequencing of the different amplicons from both ends, T7 and Sp6 primers were used. For the CS amplicon of $2000 \mathrm{bp}$, an internal primer was synthesised (based on the sequence obtained) and used to continue sequencing until the resistance genes inserted in the amplicon were identified. For isolates containing two integrons that only differed by 50 bp in size, CS-PCR products were cloned in the pGEM-T easy Vector (Promega). Plasmids with different inserts were selected on the basis of restriction enzyme analysis using EcoRI or HpaII. Purified plasmids were then used for DNA sequence determination. Dideoxy sequencing was performed on an ABI 3730 Sequencer (Applied Biosystems, Foster City, CA). DNA sequences were analysed with the Clone Manager Suit and by consulting the GenBank database of the National Center for Biotechnology Information via the BLAST network service. The nucleotide sequences of the gene cassettes have been deposited in the GenBank database under the accession numbers given in Table 2. 
Table 2.

Characterisation of class 1 integrons of Salmonella isolates in The Netherlands

\begin{tabular}{|c|c|c|c|c|c|c|c|}
\hline IP & Size in bp (isolate ID) & RE 1 & Fragments (ca. bp) & RE 2 & Fragments (ca. bp) & Gene cassette & Accession number \\
\hline \multirow[t]{2}{*}{ I } & $1000(\mathrm{~N} 216)$ & EcoRI & 550,450 & HpaII & $400,250,150,50$ & $\operatorname{aad} A 2$ & DQ133165 \\
\hline & 1200 & HincII & $700,350,150$ & HpaII & 800,400 & bla PSE-1 $_{1}$ & DQ133161 \\
\hline \multirow[t]{2}{*}{ II } & 1250 (N107) & & & & & dfrA1 & DQ123842 \\
\hline & 1200 & & & & & bla $a_{\text {PSE-1 }}$ & DQ133163 \\
\hline III & $700(\mathrm{~N} 111)$ & & & & & dfra5 & DQ133160 \\
\hline IV & $1000(\mathrm{~N} 328)$ & $B c / \mathrm{I}$ & 700,300 & HpaII & $580,240,120,60$ & $\operatorname{aad} A 1$ & DQ133159 \\
\hline $\mathrm{V}$ & $1000(\mathrm{~N} 80)$ & EcoRI & 550,450 & HpaII & $400,250,150,50$ & $\operatorname{aad} A 2$ & DQ133165 \\
\hline VI & 1200 (N209) & HincII & $700,350,150$ & HpaII & 800,400 & bla PSE-1 $_{1}$ & DQ133162 \\
\hline VII & 1500 (N133) & HincII & 1000,500 & HpaII & $700,500,200,100$ & $\operatorname{aad} A 1, \operatorname{dfr} A 1$ & DQ133164 \\
\hline VIII & 2000 (N279) & $B c / I$ & $700,650,450$ & HpaII & $700,500,300,200,100$ & $\operatorname{aad} A 2$, sat & DQ133166 \\
\hline IX & $1200(\mathrm{~N} 246)$ & & & & & dfrA14, orfC & DQ228133 \\
\hline$x$ & $-(\mathrm{N} 178)^{\mathrm{a}}$ & & & & & & \\
\hline
\end{tabular}

IP, integron profile; RE, restriction endonuclease.

${ }^{a}$ No product found from conserved segment polymerase chain reaction (CS-PCR) or inverted PCR. 


\subsection{Salmonella genomic island 1 mapping}

Integron-positive isolates were investigated for the presence of SGI1. The isolates were first examined by PCR for the presence of the left and right junction of SGI1. Next, the presence of sequences from the antibiotic resistance gene cluster was determined by PCR using primers (Table 1) described previously [12] and in this study. Template DNA was prepared with the High Pure PCR Preparation Kit (Roche, Mannheim, Germany). PCR was performed in a total volume of $25 \mu \mathrm{L}$ containing $2.5 \mu \mathrm{L}$ of $10 \times$ PCR buffer (HT Biotechnology, Cambridge, UK), $0.5 \mu \mathrm{L}$ of $10 \times$ deoxynucleotide triphosphate mix ( $2 \mathrm{mM}$ each), $50 \mathrm{pmol}$ of each primer, $1.25 \mathrm{U}$ of Taq DNA polymerase and $1 \mu \mathrm{L}$ of template DNA. To amplify fragments larger than $3.5 \mathrm{~kb}$, Taq Plus polymerase was used instead of Taq DNA polymerase (HT Biotechnology). Thermal cycling conditions consisted of a hot start cycle at $94^{\circ} \mathrm{C}$ for $3 \mathrm{~min}$, followed by 35 cycles of $1 \mathrm{~min}$ at $94^{\circ} \mathrm{C}, 1 \mathrm{~min}$ at $50-65^{\circ} \mathrm{C}$ (depending on the primers used), $1-5 \mathrm{~min}$ at $72{ }^{\circ} \mathrm{C}$ (depending on the expected amplicon size) and a final step of $72{ }^{\circ} \mathrm{C}$ for $10 \mathrm{~min}$. Annealing temperatures for primers used in the SGI 1 mapping were $50^{\circ} \mathrm{C}$ except for int1/aad, tetR/tetA $\left(54^{\circ} \mathrm{C}\right)$, sulTER/F3 and pse-L/MDR-B $\left(60^{\circ} \mathrm{C}\right)$.

\section{6. $\beta$-Lactamase production}

Ceftazidime-resistant isolates were investigated for extended-spectrum $\beta$ lactamase (ESBL) production. The isolates were also tested for TEM, SHV and CTX-M type $\beta$-lactamases by PCR amplification using the primers listed in Table 1 [25]. The expected product was sequenced and analysed according to Lahey's scheme [26]. Escherichia coli 09A488, Klebsiella pneumoniae 09A018 and Enterobacter cloacae 03773, which were previously shown to harbour TEM, SHV and CTX-M type $\beta$-lactamase, respectively, were used as positive controls.

\subsection{Statistical analysis}

All statistical analyses were performed in Microsoft Excel 2000 using $x^{2}$ tests. Differences were considered significant at $P<0.05$.

\section{Results}

In the present study, 128 isolates (54\%) were susceptible to all 15 antibiotics tested (Fig. 1). Sixty-nine Salmonella (29.1\%) were resistant to two or more antimicrobials. Antimicrobial resistance was not solely associated with a particular Salmonella serovar. Resistant isolates belonged to 25 different serovars, including Typhimurium (35/51), Dublin (10/37), Enteritidis (13/41), Virchow (8/8), Hadar, Paratyphi B (5/5), Derby (6/7), Newport (3/6), Mbandaka (3/7), Brandenburg (2/6), Senftenberg (2/7), Stanley (2/3), Livingstone (1/2), Agona (1/3), Anatum (1/3), Panama (1/4), Infantis (1/5), Albany, Blockley, Braenderup, Corvallis, Haifa, Glostrup, Indiana and London (1/1). However, resistance to antimicrobials was high (nearly 70\%) in serovar Typhimurium. Salmonella Typhimurium PT 506 (DT 104) isolates were resistant to between two and seven antimicrobials.

Resistance to sulphonamides, ampicillin, tetracycline, streptomycin, trimethoprim, nalidixic acid, chloramphenicol, cefalothin, amoxicillin/clavulanic acid, ceftazidime, kanamycin, ciprofloxacin, norfloxacin and gentamicin was found in $29 \%, 21 \%$, $17 \%, 17 \%, 13 \%, 12 \%, 9 \%, 4 \%, 2 \%, 1 \%, 0.8 \%, 0.8 \%, 0.4 \%$ and $0.4 \%$ of the isolates, respectively. 


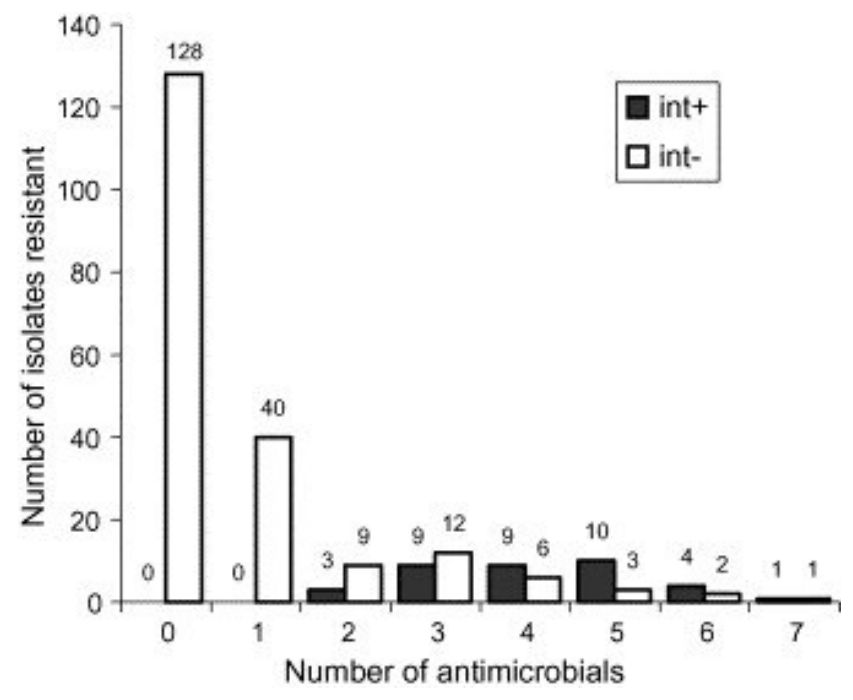

Fig. 1. Relationship between class 1 integrons and multidrug resistance $\left(X^{2}\right.$ test, $P<0.001)$.

Most isolates in the present study were susceptible to the third-generation cephalosporins. However, three $(1.3 \%)$ ceftazidime-resistant isolates (two $S$. Typhimurium from human and cattle and one $S$. Braenderup from chicken) were observed. No ESBL-producing strain was found.

Resistance to nalidixic acid was common in serovar Hadar (100\%) and serovar Virchow (87.5\%). Three isolates showing resistance to fluoroquinolones (norfloxacin (1) and ciprofloxacin (2)) and three isolates with decreased susceptibility (reduction in diameter of the inhibition zone) to these antimicrobials (norfloxacin (2) and ciprofloxacin (1)) were present. Multidrug-resistant Salmonella were obtained more frequently from animal origin $(86 \%, 50 \%, 30 \%$ and $23 \%$ of food, pig, cattle and chicken isolates, respectively) than from humans $(19 \%)$.

There was a strong relationship between multidrug resistance and the presence of class 1 integrons $(P<0.001)$ (Fig. 1$)$. Class 1 integrons were detected in 36 Salmonella isolates (15.2\%) of 11 different serovars. Integrons were found predominantly in S. Typhimurium DT $104(n=13 ; 100 \%)$. However, class 1 integrons were also identified in other phage types of $S$. Typhimurium and in other serovars (Table 3). Data analysis showed that there is a strong relationship between the presence of class 1 integrons and resistance to sulphonamides, chloramphenicol, ampicillin, tetracycline, streptomycin or trimethoprim $(P<0.001$ in order of decreasing strength of correlation), whilst resistance to other antimicrobials tested was integron independent. 
Table 3.

Resistance patterns in class 1 integron-positive Salmonella enterica isolates, their integrons and Salmonella genomic island (SGI1) types

\begin{tabular}{|c|c|c|c|c|c|}
\hline ID & Resistance profile (intermediate) & Serovar (source of isolate ${ }^{a}$ ) & Amplicon size (bp) & $\mathbf{I} \mathbf{P}^{\mathbf{b}}$ & SGI types \\
\hline N216 & AMP, CAZ, CHL, STR, TET, TMP, SUF (AMC) & Typhimurium DT $104^{\mathrm{c}}(\mathrm{H})$ & $1000 ; 1200$ & I & SGI1 \\
\hline N235 & AMP, CHL, STR, TET, TMP, SUF (AMC) & Typhimurium DT 104 (P) & $1000 ; 1200$ & I & SGI1 \\
\hline N246 & AMP, CHL, STR, TET, TMP, SUF (AMC) & Senftenberg $(\mathrm{C})$ & $1200^{d}$ & IX & - \\
\hline N193 & AMP, CHL, NAL, TET, TMP, SUF (AMC, STR) & Derby $(H)$ & $1000 ; 1200$ & I & SGI1 \\
\hline N140 & AMP, CEP, NAL, TET, TMP, SUF (AMC, STR) & Virchow (C) & 1500 & VII & - \\
\hline N94 & AMP, CHL, STR, TET, SUF (AMC) & Typhimurium DT $104(P)$ & $1000 ; 1200$ & I & SGI1 \\
\hline N176 & AMP, CHL, STR, TET, SUF (AMC) & Typhimurium DT $104(\mathrm{H})$ & $1000 ; 1200$ & I & SGI1 \\
\hline N182 & AMP, CHL, STR, TET, SUF (AMC) & Typhimurium DT $104(\mathrm{H})$ & $1000 ; 1200$ & I & SGI1 \\
\hline N188 & AMP, CHL, STR, TET, SUF & Typhimurium DT $104(\mathrm{H})$ & $1000 ; 1200$ & I & SGI1 \\
\hline N221 & AMP, CHL, STR, TET, SUF (AMC) & Typhimurium DT $104(\mathrm{H})$ & $1000 ; 1200$ & I & SGI1 \\
\hline N326 & AMP, CHL, STR, TET, SUF (AMC) & Non-DT $104^{\mathrm{e}}(\mathrm{B})$ & $1000 ; 1200$ & I & SGI1 \\
\hline N104 & AMP, CHL, NAL, STR, SUF (TET) & Typhimurium DT 104 (M) & $1000 ; 1200$ & I & SGI1 \\
\hline N167 & AMP, KAN, TET, TMP, SUF (CEP) & Non-DT 104 (M) & 2000 & VIII & - \\
\hline N203 & AMP, STR, TET, TMP, SUF & Indiana (C) & 1500 & VII & - \\
\hline
\end{tabular}




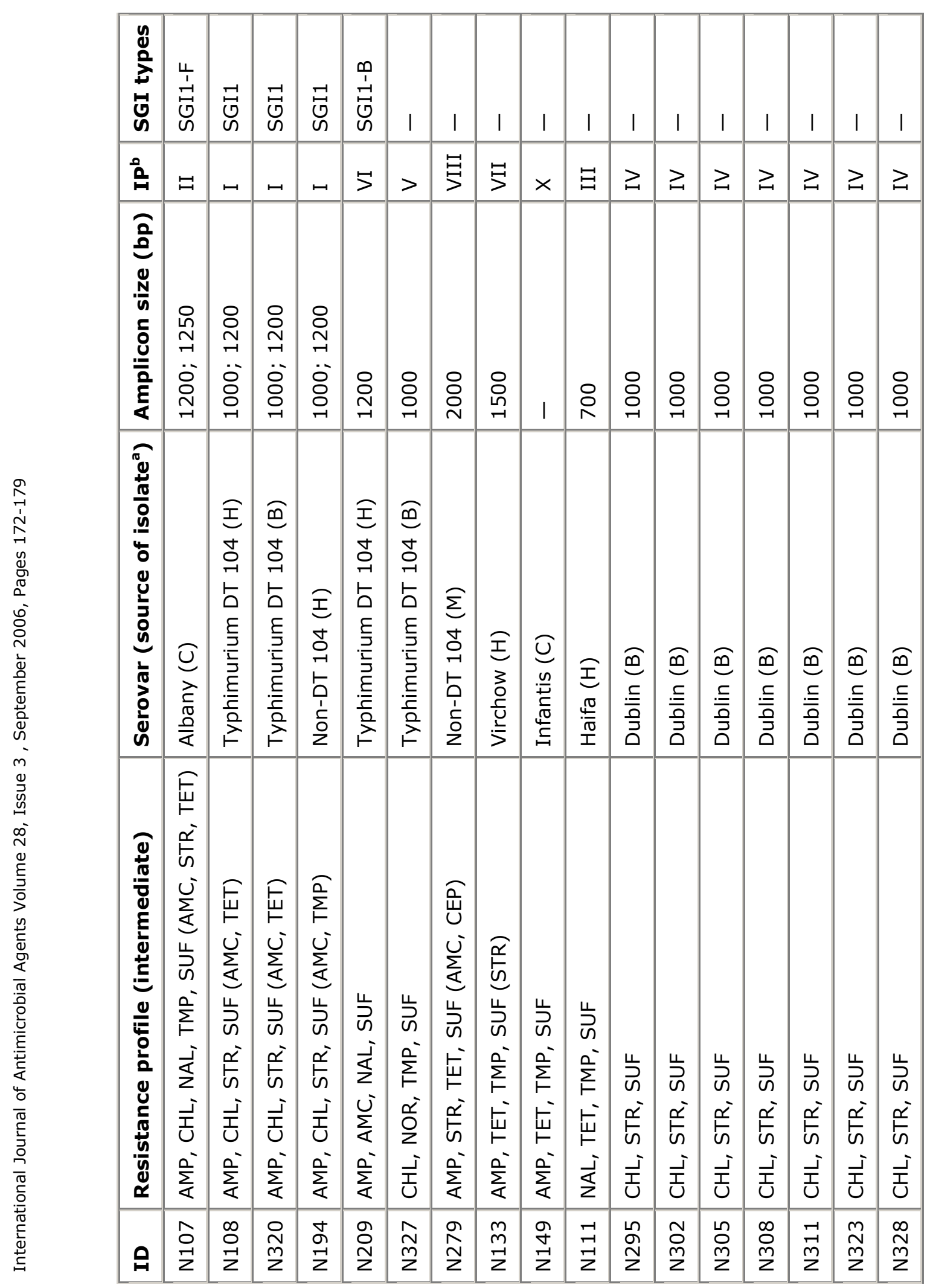




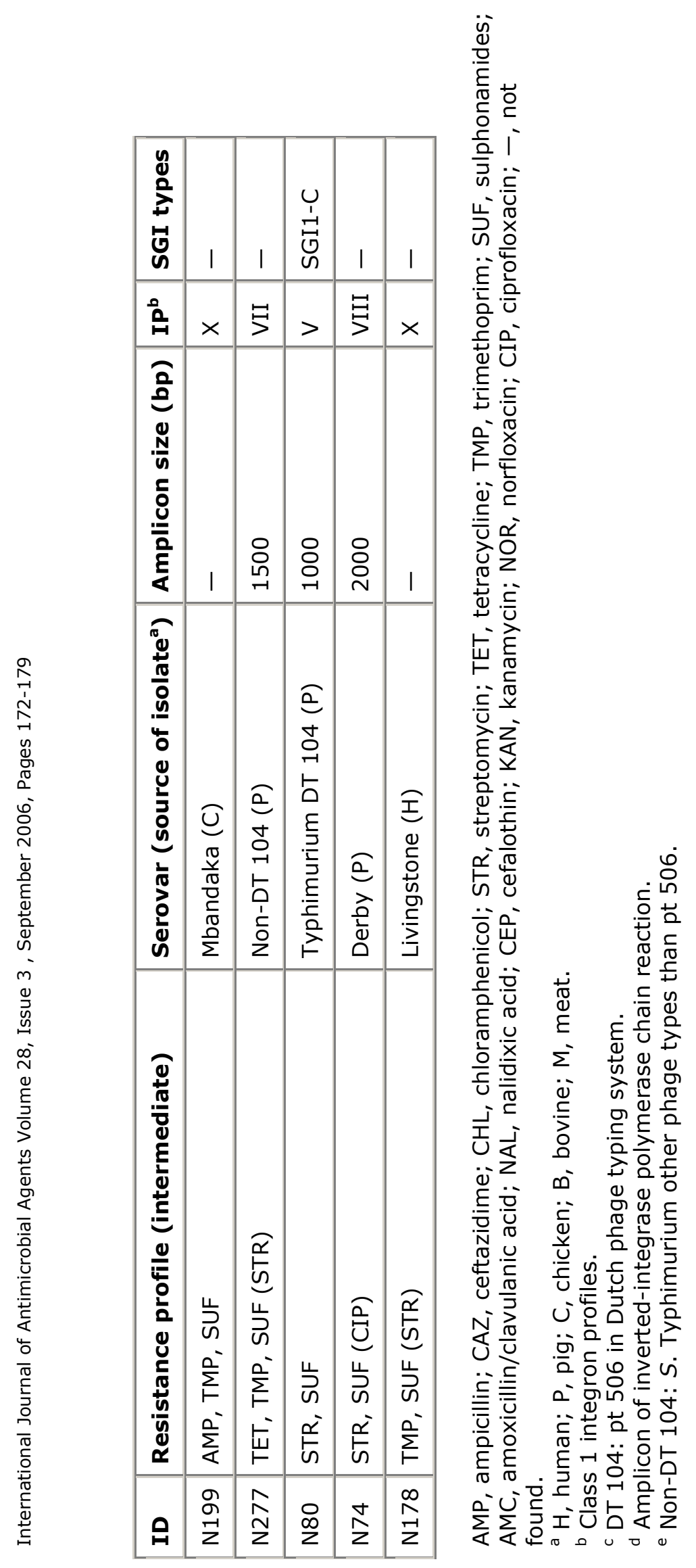


Ten class 1 integron profiles (IPs) could be defined based on the number and size of the amplicons obtained (Table 2 and Table 3). Four isolates (of serovars Infantis, Livingstone, Mbandaka and Senftenberg) were integron-positive but yielded no product in the CS-PCR. IP IX was found in S. Senftenberg using inverted PCR. IP $X$ was observed in three isolates (S. Infantis, S. Livingstone and S. Mbandaka) where no products in either CS-PCR or inverted PCR were obtained. Gene cassettes encoding resistance to streptomycin (aadA1), streptomycin and spectinomycin (aadA2), streptothricin (sat), $\beta$-lactam (bla $\mathrm{PSE}-1$ ) and trimethoprim (dfrA1, dfrA5 and dfrA14) were observed.

Complete copies of SGI1 or one of its variants were found in $16(44 \%)$ of the class 1 integron-positive isolates (Fig. 2; Table 3). SGI1 was found in 12 serovar Typhimurium and one $S$. Derby isolate. SGI1-F was present in a single S. Albany isolate. There was a strong relationship between the presence of two class 1 integrons and the presence of SGI1 $(P<0.001)$. Two other isolates of $S$. Typhimurium carrying only a single integron contained an incomplete SGI1, known as SGI1-B (strain N209) or SGI1-C (strain N80). Both yielded the expected products for the left (thdf and int genes) and right (S044 and int2 genes) junction of these SGI1s. Isolate N80 also yielded a single 1200 bp product in the amplification of the fragment between the int 1 and aadA2 genes. Isolate N209 yielded no products in the int2-pseL PCR, but a 900 bp product was generated by amplification with the int 1 and pseL primers. No products were obtained from other combined PCRs, as shown in Fig. 2, except for amplification of the $4.5 \mathrm{~kb}$ fragment from pse-1 to S044 for isolate N209. Thus, the Salmonella genomic islands in strains N209 and N80 (Table 2) were classified as SGI1-B and $-\mathrm{C}$, respectively. 


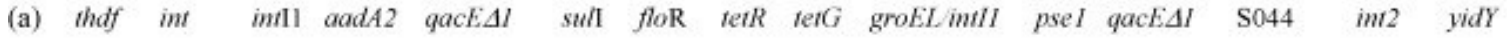

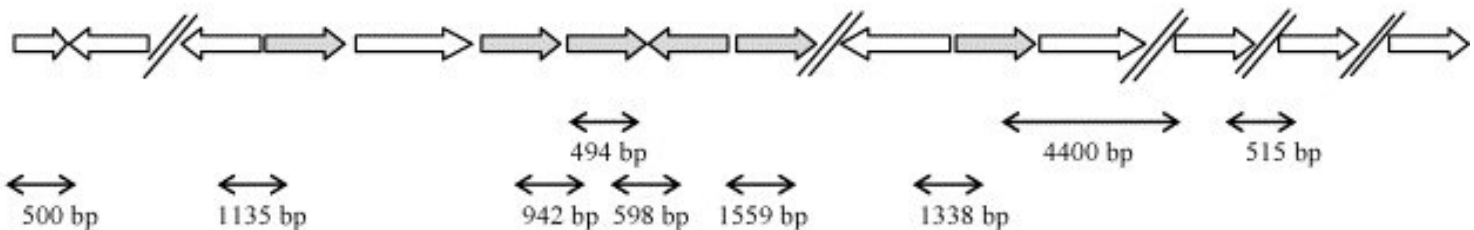

(b) thdf int intIl aadA2 qacEAl sull flo R tetR tetG groELintII psel qacEAl S044 yidY

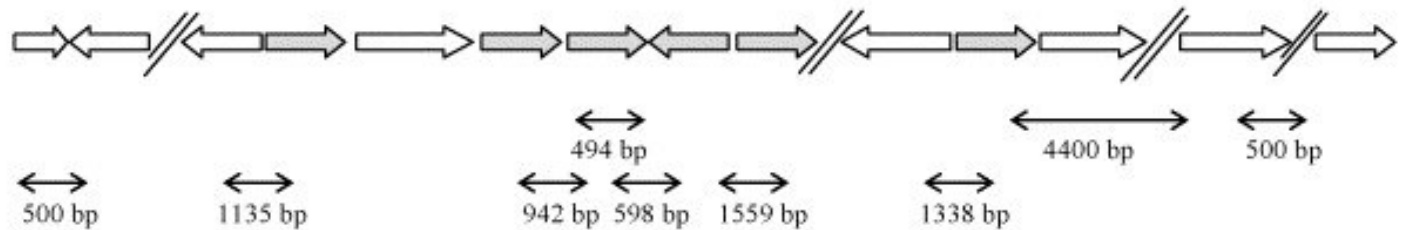

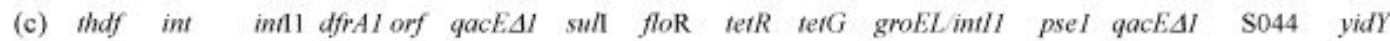

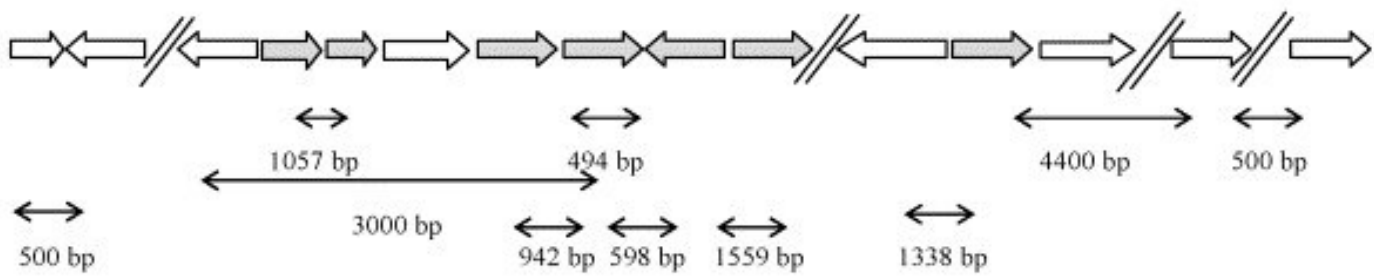

(d) thdf int infll aadA2 qacEAl sull $\mathrm{S} 044 \quad$ int2 $\quad$ yidY
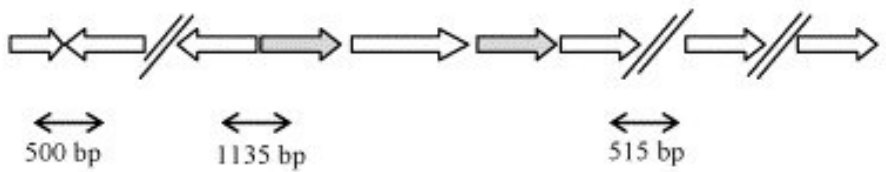

(e) thdf int intl1 pseI qacEAI sulI $\mathrm{S} 044$ int2 yidY

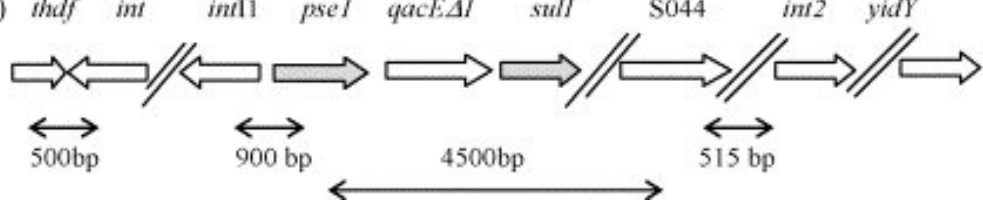

Fig. 2. Map of Salmonella genomic island 1 (SGI1) highlighting targets and fragment sizes in polymerase chain reactions used for mapping SGI1 antibiotic resistance gene clusters. (a) SGI1 found in S. Typhimurium (N216); (b) SGI1 found in S. Derby (N193); (c) SGI1-F found in S. Albany (N107); (d) SGI1-C found in S. Typhimurium (N80); and (e) SGI1-B found in S. Typhimurium (N209). The grey shades indicate resistance genes (modified from [12]).

\section{Discussion}

The main finding in this study was the widespread presence of multidrug resistance among Salmonella from human and animal origin and the diversity of class 1 integrons and SGI1 in different Salmonella serovars, including serovars in which class 1 integrons have not previously been found. Class 1 integrons have previously been detected in different Salmonella serovars including Typhimurium, Virchow, Hadar, Dublin, Derby, Haifa, Infantis, Albany and Mbandaka [27], [28] and [29]; however, to our knowledge, they have not been found in $S$. Senftenberg and $S$. Indiana. This finding suggests the spread of integrons that 
are located on a mobile genetic element among the many serovars of Salmonella. In contrast to a study from Scotland [30], no class 1 integrons were found among serovar Enteritidis in this study. In The Netherlands, antimicrobial resistance in $S$. Enteritidis isolates is still low (3\%) [31] and [32], whereas in Scotland S.

Enteritidis isolates were multiresistant and carried integrons [30]. In general, our data suggest that acquisition of integrons is not limited to specific Salmonella serovars but may occur in any serovar. Amplification of gene cassettes by CS-PCR in integrase-positive isolates is sometimes negative because the primers do not always anneal to the $3^{\prime}$-conserved segment [23]. This was also observed in the present study. An inverted-integrase PCR proved to be an effective method for the study of (at least part of) the gene cassettes present therein.

Most integron-positive serovars carried gene cassettes that have been described previously [27], [28], [33], [34] and [35]. However, neither the dfrA5 gene cassette in $S$. Haifa nor the combination of the gene cassettes aadA2 and sat in $S$. Typhimurium or dfrA14 and an unknown gene in S. Senftenberg have been reported before. The $d f r A 5$ gene has been found in $S$. Wien (accession number AY827837). The aadA2 and sat genes have been described on a plasmid of a highly invasive and resistant S. Choleraesuis strain [36]. The gene dfrA14 has only previously been observed in E. coli (accession number AJ884725) but not in Salmonella. Therefore, one may speculate that integrons or the gene cassettes they contain have been exchanged not only among Salmonella serovars but also among Enterobacteriaceae. Two class 1 integrons with sizes of ca. $1000 \mathrm{bp}$ and $1200 \mathrm{bp}$ were found in almost all S. Typhimurium DT 104 isolates, in accordance with the findings of Randall et al. [28]. In the present study, S. Typhimurium DT 104 isolates not only had a pentadrug resistance phenotype (ACSSUT), a tetradrug resistance phenotype (CSSUT) or a SSu resistance phenotype, as reported previously [27], [28], [29] and [37], but also other multiple resistance phenotypes (Table 3). These phenotypes are characteristic of SGI1 and its variants.

SGI1 is an important determinant of multidrug resistance. SGI1 or its variants with different gene cassettes located in two integrons have been found in many serovars of Salmonella enterica [11]. The high rate with which SGI1 is present in S. Typhimurium DT 104 isolates in the present and previous studies [10], [11] and [12] suggests that the SGI1 variant with the five antibiotic resistance genes aadA2, sul1, floR, tet and pse is the oldest and probably the first to arise. Its variants in other Salmonella serovars [11] probably arose as the result of recombination events. Deletion is a possible explanation for the structure of SGI1-B and SGI1-C in the two S. Typhimurium isolates that contain only one integron flanked by the left and right junction of SGI1. The fact that PCR products for isolate N209 could only be obtained with int1-pseL primers but not with int2pseL primers suggests another location for the bla $a_{\text {PSE- } 1}$ gene at the left junction of SGI1 owing to a single crossover event at intI1 [9]. The genes found adjacent to the left junction of SGI1, such as the int and xis genes, could have an important role in the excision and integration of integron-located gene cassettes [14]. In this study, the expression of the tetracycline resistance gene (tetG) varied from full resistance among SGI1-carrying $S$. Typhimurium isolates to intermediate susceptibility in an SGI1-F-positive S. Albany isolate. Since a promoter is not included in the cassette, the level of antibiotic resistance expressed by the cassette gene is influenced by the position of the cassette in the array if more than one cassette is present. In all cases the resistance level was highest when the gene was closest to the promoter [38], which is located in the $5^{\prime}$-conserved segment of the class 1 integron [5]. 
In summary, multidrug resistance was strongly associated with the presence of class 1 integrons in Salmonella. We found gene cassettes or combinations of gene cassettes not previously reported in integrons in Salmonella. Class 1 integrons were detected for the first time in serovars Indiana and Senftenberg. SGI1 and its variants (SGI1-B, $-C$ and $-F$ ) were found in serovars Typhimurium, Derby and Albany. Similar resistance patterns, integron types and SGI1 structures were found regardless of the source of the isolate (human or animal).

\section{Acknowledgments}

This study was funded by a grant of the Vietnamese government. We thank Max Heck and Frans Bensink of RIVM; Marcel de Zoete of I\&I for technical help; and Armand Paauw of Eijkman-Winkler Institute for providing control strains.

\section{References}

[1] W. van Pelt, M.A. de Wit, W.J. Wannet, E.J. Ligtvoet, M.A. Widdowson and Y.T. van Duynhoven, Laboratory surveillance of bacterial gastroenteric pathogens in The Netherlands, 1991-2001, Epidemiol Infect 130 (2003), pp. 431-441.

[2] T. Humphrey, Public health aspects of Salmonella infections. In: C. Wray and A. Wray, Editors, Salmonella in domestic animals, CABI Publishing, New York, NY (2000), pp. 245-263.

[3] Food and Agriculture Organization of the United Nations/World Health Organization/World Organization for Animal Health. Joint FAO/OIE/WHO Expert Workshop on non-human antimicrobial usage and antimicrobial resistance: scientific assessment.

http://www.who.int/foodsafety/publications/micro/en/amr.pdf [accessed 4 July 2006].

[4] M.A. Leverstein-van Hall, H.E.M. Blok, A.R.T. Donders, A. Paauw, A.C. Fluit and J. Verhoef, Multidrug resistance among Enterobacteriaceae is strongly associated with the presence of integrons and is independent of species or isolate origin, J Infect Dis 187 (2003), pp. 251-259.

[5] R.M. Hall and H.W. Stokes, Integrons: novel DNA elements which capture genes by site-specific recombination, Genetica 90 (1993), pp. 115-132.

[6] H.W. Stokes and R.M. Hall, A novel family of potentially mobile DNA elements encoding sitespecific gene-integration functions: integrons, Mol Microbiol 3 (1989), pp. 1669-1683.

[7] A.C. Fluit, Towards more virulent and antibiotic-resistant Salmonella?, FEMS Immunol Med Microbiol 43 (2005), pp. 1-11.

[8] P. Ebner, K. Garner and A. Mathew, Class 1 integrons in various Salmonella enterica serovars isolated from animals and identification of genomic island SGI1 in Salmonella enterica var, Meleagridis. J Antimicrob Chemother 53 (2004), pp. 1004-1009.

[9] D. Boyd, A. Cloeckaert, E. Chaslus-Dancla and M.R. Mulvey, Characterization of variant Salmonella genomic island 1 multidrug resistance regions from serovars Typhimurium DT104 and Agona, Antimicrob Agents Chemother 46 (2002), pp. 1714-1722.

[10] D. Meunier, D. Boyd and M.R. Mulvey et al., Salmonella enterica serotype Typhimurium DT 104 antibiotic resistance genomic island I in serotype paratyphi B, Emerg Infect Dis 8 (2002), pp. 430433.

[11] R.S. Levings, D. Lightfoot, S.R. Partridge, R.M. Hall and S.P. Djordjevic, The genomic island SGI1, containing the multiple antibiotic resistance region of Salmonella enterica serovar Typhimurium DT104 or variants of it, is widely distributed in other S. enterica serovars, J Bacteriol 187 (2005), pp. 4401-4409. 
[12] B. Doublet, R. Lailler and D. Meunier et al., Variant Salmonella genomic island 1 antibiotic resistance gene cluster in Salmonella enterica serovar Albany, Emerg Infect Dis 9 (2003), pp. 585591.

[13] B. Doublet, D. Boyd, M.R. Mulvey and A. Cloeckaert, The Salmonella genomic island 1 is an integrative mobilizable element, Mol Microbiol 55 (2005), pp. 1911-1924.

[14] D. Boyd, G.A. Peters and A. Cloeckaert et al., Complete nucleotide sequence of a 43-kilobase genomic island associated with the multidrug resistance region of Salmonella enterica serovar Typhimurium DT104 and its identification in phage type DT120 and serovar Agona, J Bacteriol 183 (2001), pp. 5725-5732.

[15] M.A. Arcangioli, S. Leroy-Setrin, J.L. Martel and E. Chaslus-Dancla, A new chloramphenicol and florfenicol resistance gene flanked by two integron structures in Salmonella Typhimurium DT104, FEMS Microbiol Lett 174 (1999), pp. 327-332.

[16] C.E. Briggs and P.M. Fratamico, Molecular characterization of an antibiotic resistance gene cluster of Salmonella Typhimurium DT104, Antimicrob Agents Chemother 43 (1999), pp. 846-849.

[17] B. Doublet, P. Butaye and H. Imberechts et al., Salmonella genomic island 1 multidrug resistance gene clusters in Salmonella enterica serovar Agona isolated in Belgium in 1992 to 2002, Antimicrob Agents Chemother 48 (2004), pp. 2510-2517.

[18] P.R. Edwards and W.H. Ewing, Edwards and Ewing's identification of Enterobacteriaceae (4th ed.), Elsevier Science Publishing Co. Inc., New York, NY (1986).

[19] M.Y. Popoff, Antigenic formulas of the Salmonella serovars (8th ed.), WHO Collaborating Center for Reference and Research on Salmonella, Paris, France (2001).

[20] L.R. Ward, J.D. de Sa and B. Rowe, A phage-typing scheme for Salmonella Enteritidis, Epidemiol Infect 99 (1987), pp. 291-294.

[21] P.A. Guinee, W.J. van Leeuwen and D. Pruys, Phage typing of S. Typhimurium in The Netherlands. 1. The phage typing system, Zentralbl Bakteriol 226 (1974), pp. 194-200.

[22] National Committee for Clinical Laboratory Standards, Performance standards for antimicrobial disk and dilution susceptibility tests. M31-A2. Approved standard (2nd ed.), NCCLS, Wayne, PA (2001).

[23] C. Levesque, L. Piche, C. Larose and P.H. Roy, PCR mapping of integrons reveals several novel combinations of resistance genes, Antimicrob Agents Chemother 39 (1995), pp. 185-191.

[24] M.A. Leverstein-Van Hall, A. Paauw, A.T. Box, H.E. Blok, J. Verhoef and A.C. Fluit, Presence of integron-associated resistance in the community is widespread and contributes to multidrug resistance in the hospital, J Clin Microbiol 40 (2002), pp. 3038-3040.

[25] P.A. Bradford, Automated thermal cycling is superior to traditional methods for nucleotide sequencing of bla $\mathrm{sHv}_{\mathrm{siv}}$ genes, Antimicrob Agents Chemother 43 (1999), pp. 2960-2963.

[26] Lahey Clinic. Amino acid sequences for TEM, SHV, and OXA extended-spectrum and inhibitor resistant beta-lactamases. http://www.lahey.org/Studies/ [accessed 4 July 2006].

[27] B. Guerra, S. Soto, S. Cal and M.C. Mendoza, Antimicrobial resistance and spread of class 1 integrons among Salmonella serotypes, Antimicrob Agents Chemother 44 (2000), pp. 2166-2169.

[28] L.P. Randall, S.W. Cooles, M.K. Osborn, L.J. Piddock and M.J. Woodward, Antibiotic resistance genes, integrons and multiple antibiotic resistance in thirty-five serotypes of Salmonella enterica isolated from humans and animals in the UK, J Antimicrob Chemother 53 (2004), pp. 208-216.

[29] D.G. White, S. Zhao and P.F. McDermott et al., Characterization of integron mediated antimicrobial resistance in Salmonella isolated from diseased swine, Can J Vet Res 67 (2003), pp. 3947. 
[30] A.W. Brown, S.C. Rankin and D.J. Platt, Detection and characterisation of integrons in Salmonella enterica serotype Enteritidis, FEMS Microbiol Lett 191 (2000), pp. 145-149.

[31] E. van Duijkeren, W.J. Wannet, D.J. Houwers and W. van Pelt, Antimicrobial susceptibilities of Salmonella strains isolated from humans, cattle, pigs, and chickens in The Netherlands from 1984 to 2001, J Clin Microbiol 41 (2003), pp. 3574-3578.

[32] Mevius DJ, van Pelt W, editors. MARAN 2002. Monitoring of antimicrobial resistance and antibiotic usage in animals in The Netherlands in 2003. (http://www.cidclelystad.wur.nl/NR/rdonlyres/B281959D-AC66-4326-9C86-

35364655E5A6/11378/MARAN2002web4.pdf) [accessed 4 July 2006].

[33] M. Daly and S. Fanning, Characterization and chromosomal mapping of antimicrobial resistance genes in Salmonella enterica serotype Typhimurium, Appl Environ Microbiol 66 (2000), pp. $4842-$ 4848.

[34] A. del Cerro, S.M. Soto and M.C. Mendoza, Virulence and antimicrobial-resistance gene profiles determined by PCR-based procedure for Salmonella isolated from samples of animal origin, Food Microbiol 20 (2003), pp. 431-438.

[35] S. Zhao, A.R. Datta, S. Ayers, S. Friedman, R.D. Walker and D.G. White, Antimicrobial-resistant Salmonella serovars isolated from imported foods, Int J Food Microbiol 84 (2003), pp. 87-92.

[36] C.H. Chiu, P. Tang and C. Chu et al., The genome sequence of Salmonella enterica serovar Choleraesuis, a highly invasive and resistant zoonotic pathogen, Nucleic Acids Res 33 (2005), pp. 1690-1698.

[37] W.A. Gebreyes and C. Altier, Molecular characterization of multidrug-resistant Salmonella enterica subsp. enterica serovar Typhimurium isolates from swine, J Antimicrob Chemother $\mathbf{4 0}$ (2002), pp. 2813-2822.

[38] C.M. Collis and R.M. Hall, Expression of antibiotic resistance genes in the integrated cassettes of integrons, Antimicrob Agents Chemother 39 (1995), pp. 155-162.

* Corresponding author. Tel.: +3130253 4888; fax: +31302540784. 